\title{
On Solutions of a Nonlinear Erdélyi-Kober Integral Equation
}

\author{
Nurgali K. Ashirbayev, ${ }^{1}$ Józef Banaś, ${ }^{2}$ and Raina Bekmoldayeva ${ }^{1}$ \\ ${ }^{1}$ Department of Mathematics, Science-Pedagogical Faculty, M. Auezov South Kazakhstan State University, \\ Tauke Khan Avenue 5, Shymkent 160012, Kazakhstan \\ ${ }^{2}$ Department of Mathematics, Rzeszów University of Technology, Al. Powstańców Warszawy 8, 35-959 Rzeszów, Poland
}

Correspondence should be addressed to Józef Banaś; jbanas@prz.edu.pl

Received 27 April 2014; Accepted 18 May 2014; Published 1 June 2014

Academic Editor: Robert A. Van Gorder

Copyright (c) 2014 Nurgali K. Ashirbayev et al. This is an open access article distributed under the Creative Commons Attribution License, which permits unrestricted use, distribution, and reproduction in any medium, provided the original work is properly cited.

We conduct some investigations concerning the solvability of a nonlinear integral equation of Erdélyi-Kober type. To facilitate our study we will first consider a nonlinear integral equation of Volterra-Stieltjes type. Since the mentioned Erdélyi-Kober integral equation turns out to be a special case of that of Volterra-Stieltjes type, we can apply the obtained results to the Erdélyi-Kober integral equation. Examples illustrating the obtained results will be also included.

\section{Introduction}

Differential and integral operators of noninteger order play an important role in several branches of applied mathematics, engineering, physics, mathematical physics, and so forth. Differential and integral equations involving operators of the mentioned type are called equations of fractional order (see [1-4], e.g.). Such equations are used to describe nonlinear oscillations of earthquakes, the fluid dynamic traffic model, and the media with fractional mass dimension and they are used in the theory of viscoelasticity as well as in electrochemistry, for instance [1-8]. It is also worthwhile mentioning that differential and integral equations are also applied in biology and economics $[2,4,7]$.

Our aim in this paper is to study the solvability of the so-called nonlinear integral equations of Erdélyi-Kober type. Let us mention that integral equations of that type can be utilized in describing the generalized grey Brownian motion as a diffusion process. Other examples of integral equations of Erdélyi-Kober type are indicated in [9], for example (cf. also [10-12]).

The approach which will be used in this paper to study nonlinear integral equations of Erdélyi-Kober integral equations depends on considering some class of Volterra-Stieltjes integral equations of such a type that the mentioned ErdélyiKober integral equations turn out to be special cases of those integral equations of Volterra-Stieltjes type. Such an approach allows us not only to conduct our investigations in a more convenient way but also to obtain more general results.

\section{Notation, Definitions, and Auxiliary Facts}

In this section we collect notation, definitions, and auxiliary facts which will be utilized in our further considerations. Throughout the paper we will denote by $\mathbb{R}$ the set of real numbers and by $\mathbb{R}_{+}$the interval $[0, \infty)$. If $E$ is a Banach space with the norm $\|\cdot\|$ and the zero element $\theta$, then $B(x, r)$ will denote closed ball centered at $x$ and with radius $r$. We write $B_{r}$ to denote the ball $B(\theta, r)$.

The investigations of this paper will be conducted in the classical Banach function space $C(I)$, where $I=[0,1]$ is the unit interval. Obviously, the interval $[0,1]$ can be replaced by any interval $[a, b]$. We will assume that the space $C(I)$ is endowed with the standard maximum norm $\|\cdot\|$; that is, $\|x\|=\max \{|x(t)|: t \in I\}$.

If $x$ is an arbitrary function from the space $C(I)$, then the symbol $\omega(x, \varepsilon)$ will denote the modulus of continuity of the function $x$ defined in the standard way:

$$
\omega(x, \varepsilon)=\sup \{|x(t)-x(s)|: t, s \in I,|t-s| \leq \varepsilon\},
$$

for any $\varepsilon \geq 0$. 
Further, we recall some facts concerning functions of bounded variation [13].

At the beginning let us assume that $x$ is a real function defined on a given interval $[a, b]$. Then, by the symbol $\bigvee_{a}^{b} x$ we will denote the variation of the function $x$ on the interval $[a, b]$. If the variation $\bigvee_{a}^{b} x$ is finite, we say that $x$ is of bounded variation on the interval $[a, b]$. In the case when $u(t, s)=u$ : $[a, b] \times[c, d] \rightarrow \mathbb{R}$ and $[p, q]$ is an arbitrary subinterval of the interval $[a, b]$, we denote by $\bigvee_{t=p}^{q} u(t, s)$ the variation of the function $t \rightarrow u(t, s)$ on the interval $[p, q]$, where $s$ is a fixed number in the interval $[c, d]$. Similarly we define the quantity $\bigvee_{s=p}^{q} u(t, s)$.

Other facts concerning functions of bounded variation may be found in [13].

If $x$ and $\varphi$ are two real functions defined on the interval $[a, b]$, then under some additional conditions $[13,14]$ we can define the Stieltjes integral (in the Riemann-Stieltjes sense)

$$
\int_{a}^{b} x(t) d \varphi(t)
$$

of the function $x$ with respect to the function $\varphi$. In such a situation we will say that $x$ is Stieltjes integrable on the interval $[a, b]$ with respect to the function $\varphi$.

Let us notice that several conditions are known which guarantee the Stieltjes integrability [13-15]. One of the most frequently used requires that $x$ be continuous and $\varphi$ be of bounded variation on $[a, b]$.

Further on, we will apply a few properties of the Stieltjes integral contained in formulated lemmas below [13].

Lemma 1. If $x$ is Stieltjes integrable on the interval $[a, b]$ with respect to a function $\varphi$ of bounded variation, then

$$
\left|\int_{a}^{b} x(t) d \varphi(t)\right| \leq \int_{a}^{b}|x(t)| d\left(\bigvee_{a}^{t} \varphi\right)
$$

Lemma 2. Let $x_{1}, x_{2}$ be Stieltjes integrable functions on the interval $[a, b]$ with respect to a nondecreasing function $\varphi$, such that $x_{1}(t) \leq x_{1}(t)$ for $t \in[a, b]$. Then,

$$
\int_{a}^{b} x_{1}(t) d \varphi(t) \leq \int_{a}^{b} x_{2}(t) d \varphi(t) .
$$

In the sequel we will also consider the Stieltjes integrals having the form

$$
\int_{a}^{b} x(s) d_{s} g(t, s),
$$

where $g:[a, b] \times[a, b] \rightarrow \mathbb{R}$ and the symbol $d_{s}$ indicates the integration with respect to $s$. The details concerning the integral of such a kind will be given later. Let us only mention that integral (5) allows us to represent the Erdélyi-Kober integral equation in the more convenient form as a particular case of the Volterra-Stieltjes integral equation (cf. the next section).

\section{Introductory Facts concerning Erdélyi-Kober Integral Equations}

In this section we provide a few preliminary facts concerning the integral equations of Erdélyi-Kober type. The theory of those equations was initiated by the papers of Erdélyi and Kober [16-18].

Integral equations of Erdélyi-Kober type can be presented in the form of singular nonlinear integral equations having the form:

$$
x(t)=a(t)+\frac{1}{\Gamma(\alpha)} \int_{0}^{t} \frac{s^{\gamma m} f(t, s, x(s))}{\left(t^{m}-s^{m}\right)^{1-\alpha}} d s,
$$

where $m, \gamma, \alpha$ are positive constants, $\alpha \in(0,1)$, and $t \in I=$ $[0,1]$. Moreover, $\Gamma(\alpha)$ is the gamma function.

This equation can be written in a more general form:

$$
x(t)=a(t)+\frac{t^{-m(\gamma+\alpha)}}{\Gamma(\alpha)} \int_{0}^{t} \frac{s^{\gamma m} f(t, s, x(s))}{\left(t^{m}-s^{m}\right)^{1-\alpha}} d s
$$

(cf. [12]), but the function $t^{-m(\gamma+\alpha)}$ can be linked with the function $f(t, s, x(s))$ and such an approach allows us to convert (7) to the form (6).

Notice that taking $\gamma=1-1 / m$ (for $m \geq 1$ ), we transform (6) to the form

$$
x(t)=a(t)+\frac{1}{\Gamma(\alpha)} \int_{0}^{t} \frac{s^{m-1} f(t, s, x(s))}{\left(t^{m}-s^{m}\right)^{1-\alpha}} d s,
$$

so we obtain (with the accuracy to the constant $m$ ) the Erdélyi-Kober integral equation of the form

$$
x(t)=a(t)+\frac{1}{\Gamma(\alpha)} \int_{0}^{t} \frac{m s^{m-1} f(t, s, x(s))}{\left(t^{m}-s^{m}\right)^{\alpha-1}} d s .
$$

In what follows we will consider (6) which will be written in the form

$$
x(t)=a(t)+\frac{1}{\Gamma(\alpha)} \int_{0}^{t} \frac{m s^{m-1} s^{\gamma m-m+1} f(t, s, x(s))}{\left(t^{m}-s^{m}\right)^{1-\alpha}} d s .
$$

In order to simplify the investigations we will assume that $\gamma>1-1 / m$ since in such a case we have that $\gamma m-m+1>0$.

Particularly, this condition is satisfied for $m \in(0,1]$. Under such an assumption the above equation can be considered in a more handy form as follows:

$$
x(t)=a(t)+\frac{1}{\Gamma(\alpha)} \int_{0}^{t} \frac{m s^{m-1} s^{p} f(t, s, x(s))}{\left(t^{m}-s^{m}\right)^{1-\alpha}} d s,
$$

where $p=$ const, $p>0$, and $t \in I$.

It is a key point in our considerations to observe that the fractional order integral equation of Erdélyi-Kober type (11) can be written as the integral equation of Volterra-Stieltjes type having the form

$$
x(t)=a(t)+\frac{\alpha}{\Gamma(\alpha)} \int_{0}^{t} s^{p} f(t, s, x(s)) d_{s} g(t, s),
$$


where the function $g=g(t, s)$ appearing in our equation has the form

$$
g(t, s)=t^{\alpha m}-\left(t^{m}-s^{m}\right)^{\alpha},
$$

for $(t, s) \in \Delta=\{(t, s): 0 \leq s \leq t \leq 1\}$.

Indeed, we have

$$
\begin{aligned}
d_{s} g(t, s) & =\frac{\partial}{\partial s} g(t, s) d s=(-\alpha)\left(t^{m}-s^{m}\right)^{\alpha-1}(-m) s^{m-1} d s \\
& =\alpha\left(t^{m}-s^{m}\right)^{\alpha-1} m s^{m-1} d s \\
& =\alpha \frac{m s^{m-1}}{\left(t^{m}-s^{m}\right)^{1-\alpha}} d s .
\end{aligned}
$$

Hence we see that nonlinear Erdélyi-Kober integral equation (11) is a particular case of nonlinear Volterra-Stieltjes integral equation (12).

This observation will be very essential in our further investigations which will be conducted in next sections.

\section{Main Result}

This section is devoted to the study of the nonlinear VolterraStieltjes integral equation (12). In our investigations we will impose the below formulated hypotheses (cf. [19]).

(i) $a \in C(I)$.

(ii) The function $g(t, s)=g: \Delta \rightarrow \mathbb{R}$ is continuous on the triangle $\Delta=\{(t, s): 0 \leq s \leq t \leq 1\}$.

(iii) The function $s \rightarrow g(t, s)$ is of bounded variation on the interval $[0, t]$, for each fixed $t \in I$.

(iv) For any $\varepsilon>0$, there exists $\delta>0$ such that, for all $t_{1}$, $t_{2} \in I$ with $t_{1}<t_{2}$ and $t_{2}-t_{1} \leq \delta$, the following inequality holds:

$$
\bigvee_{s=0}^{t_{1}}\left[g\left(t_{2}, s\right)-g\left(t_{1}, s\right)\right] \leq \varepsilon .
$$

(v) $g(t, 0)=0$ for any $t \in I$.

(vi) $f: \Delta \times \mathbb{R} \rightarrow \mathbb{R}$ is continuous and such that $|f(t, s, x)| \leq \phi(|x|)$, for all $(t, s) \in \Delta$ and for each $x \in \mathbb{R}$, where $\phi: \mathbb{R}_{+} \rightarrow \mathbb{R}_{+}$is a nondecreasing function.

In order to formulate our last assumption we will need to recall a few auxiliary facts coming from [19] and concerning the function $g=g(t, s)$ (cf. also [20]).

We will assume that $g$ satisfies assumptions (ii)-(v) formulated above.

We start with the following lemma.

Lemma 3. The function

$$
q \longrightarrow \bigvee_{s=0}^{q} g(t, s)
$$

is continuous on the interval $[0, t]$ for any fixed $t \in I$.
Lemma 4. Let assumptions (ii)-(iv) be satisfied. Then, for an arbitrarily fixed number $t_{2} \in I\left(t_{2}>0\right)$ and for any $\varepsilon>0$, there exists $\delta>0$ such that if $t_{1} \in I, t_{1}<t_{2}$ and $t_{2}-t_{1} \leq \delta$, then

$$
\bigvee_{s=t_{1}}^{t_{2}} g\left(t_{2}, s\right) \leq \varepsilon
$$

Lemma 5. Under assumptions (ii)-(iv), the function

$$
t \longrightarrow \bigvee_{s=0}^{t} g(t, s)
$$

is continuous on the interval I.

Further, let us observe that based on Lemma 5 we infer that there exists a positive constant $K$ such that

$$
K=\sup \left\{\bigvee_{s=0}^{t} g(t, s): t \in I\right\}
$$

Now, we are prepared to formulate our last assumption. (vii) There exists a positive solution $r_{0}$ of the inequality

$$
\|a\|+\frac{\alpha}{\Gamma(\alpha)} K \phi(r) \leq r .
$$

The main result of the paper is contained in the formulated theorem below.

Theorem 6. Under assumptions (i)-(vii) there exists at least one solution of (12) belonging to the ball $B_{r_{0}}$ of the space $C(I)$.

Proof. Let us consider the operator $F$ defined on the space $C(I)$ in the following way:

$$
(F x)(t)=a(t)+\frac{\alpha}{\Gamma(\alpha)} \int_{0}^{t} s^{p} f(t, s, x(s)) d_{s} g(t, s) .
$$

for $x \in C(I)$ and for arbitrarily fixed $t \in I$.

Then, taking into account the imposed assumptions we infer that the function $F x$ is well defined.

For further purposes, to make our proof more transparent, we introduce two functions $M=M(\varepsilon)$ and $N=N(\varepsilon)$ defined in the following way:

$$
\begin{gathered}
M(\varepsilon)=\sup \left\{\bigvee_{s=0}^{t_{1}}\left[g\left(t_{2}, s\right)-g\left(t_{1}, s\right)\right]: t_{1}, t_{2} \in I,\right. \\
\left.t_{1}<t_{2}, t_{2}-t_{1} \leq \varepsilon\right\}, \\
N(\varepsilon)=\sup \left\{\bigvee_{s=t_{1}}^{t_{2}} g\left(t_{2}, s\right): t_{1}, t_{2} \in I, t_{1}<t_{2}, t_{2}-t_{1} \leq \varepsilon\right\} .
\end{gathered}
$$

Observe that in virtue of assumption (iv) and Lemma 4 we conclude easily that $M(\varepsilon) \rightarrow 0$ and $N(\varepsilon) \rightarrow 0$ as $\varepsilon \rightarrow 0$. 
Now, choose arbitrarily a function $x \in C(I)$ and number $\varepsilon>0$. Next, fix $t_{1}, t_{2} \in I$ such that $\left|t_{2}-t_{1}\right| \leq \varepsilon$. Without loss of generality we can assume that $t_{1}<t_{2}$. Then, keeping in mind our assumptions and Lemmas 1 and 2, we have

$$
\begin{aligned}
& \left|(F x)\left(t_{2}\right)-(F x)\left(t_{1}\right)\right| \\
& \leq\left|a\left(t_{2}\right)-a\left(t_{1}\right)\right| \\
& +\frac{\alpha}{\Gamma(\alpha)} \mid \int_{0}^{t_{2}} s^{p} f\left(t_{2}, s, x(s)\right) d_{s} g\left(t_{2}, s\right) \\
& -\int_{0}^{t_{1}} s^{p} f\left(t_{1}, s, x(s)\right) d_{s} g\left(t_{1}, s\right) \mid \\
& \leq \omega(a, \varepsilon)+\frac{\alpha}{\Gamma(\alpha)} \mid \int_{0}^{t_{2}} s^{p} f\left(t_{2}, s, x(s)\right) d_{s} g\left(t_{2}, s\right) \\
& +\frac{\alpha}{\Gamma(\alpha)} \mid \int_{0}^{t_{2}} s^{p} f\left(t_{1}, s, x(s)\right) d_{s} g\left(t_{2}, s\right) \\
& \quad-\int_{0}^{t_{1}} s^{p} f\left(t_{1}, s, x(s)\right) d_{s} g\left(t_{2}, s\right) \mid \\
& +\frac{\alpha}{\Gamma(\alpha)} \mid \int_{0}^{t_{1}} s^{p} f\left(t_{1}, s, x(s)\right) d_{s} g\left(t_{2}, s\right) \\
& \quad \int_{0}^{t_{1}} s^{p} f\left(t_{1}, s, x(s)\right) d_{s} g\left(t_{1}, s\right) \mid
\end{aligned}
$$

$\leq \omega(a, \varepsilon)$

$$
\begin{aligned}
& +\frac{\alpha}{\Gamma(\alpha)} \mid \int_{0}^{t_{2}} s^{p}\left[f\left(t_{2}, s, x(s)\right)-f\left(t_{1}, s, x(s)\right)\right] d_{s} \\
& \quad \times g\left(t_{2}, s\right) \mid \\
& +\frac{\alpha}{\Gamma(\alpha)}\left|\int_{t_{1}}^{t_{2}} s^{p} f\left(t_{1}, s, x(s)\right) d_{s} g\left(t_{s}, s\right)\right| \\
& +\frac{\alpha}{\Gamma(\alpha)}\left|\int_{0}^{t_{1}} s^{p} f\left(t_{1}, s, x(s)\right) d_{s}\left[g\left(t_{2}, s\right)-g\left(t_{1}, s\right)\right]\right| \\
& +\omega(a, \varepsilon) \\
& +\frac{\alpha}{\Gamma(\alpha)} \int_{0}^{t_{2}} s^{p}\left|f\left(t_{2}, s, x(s)\right)-f\left(t_{1}, s, x(s)\right)\right| d_{s} \\
& +\frac{\alpha}{\Gamma(\alpha)} \int_{0}^{t_{1}} s^{p}\left|f\left(t_{1}, s, x(s)\right)\right| d_{s} \\
& +\frac{\alpha}{\Gamma(\alpha)} \int_{t_{1}}^{t_{2}} s^{p}\left|f\left(t_{1}, s, x(s)\right)\right| d_{s}\left(\bigvee_{q=0}^{s} g\left(t_{2}, q\right)\right)
\end{aligned}
$$

$$
\begin{aligned}
& \times\left(\bigvee_{q=0}^{s}\left[g\left(t_{2}, q\right)-g\left(t_{1}, q\right)\right]\right) \\
& \leq \omega(a, \varepsilon)+\frac{\alpha}{\Gamma(\alpha)} \int_{0}^{t_{2}} s^{p} \omega_{\|x\|}^{1}(f, \varepsilon) d_{s}\left(\bigvee_{q=0}^{s} g\left(t_{2}, q\right)\right) \\
& +\frac{\alpha}{\Gamma(\alpha)} \int_{t_{1}}^{t_{2}} s^{p} \phi(|x(s)|) d_{s}\left(\bigvee_{q=0}^{s} g\left(t_{2}, q\right)\right) \\
& +\frac{\alpha}{\Gamma(\alpha)} \int_{0}^{t_{1}} s^{p} \phi(|x(s)|) d_{s}\left(\bigvee_{q=0}^{s}\left[g\left(t_{2}, q\right)-g\left(t_{1}, q\right)\right]\right) \\
& \leq \omega(a, \varepsilon)+\frac{\alpha}{\Gamma(\alpha)} \omega_{\|x\|}^{1}(f, \varepsilon) \int_{0}^{t_{2}} d_{s}\left(\bigvee_{q=0}^{s} g\left(t_{2}, q\right)\right) \\
& +\frac{\alpha}{\Gamma(\alpha)} \phi(\|x\|) \int_{t_{1}}^{t_{2}} d_{s}\left(\bigvee_{q=0}^{s} g\left(t_{2}, q\right)\right) \\
& +\frac{\alpha}{\Gamma(\alpha)} \phi(\|x\|) \int_{0}^{t_{1}} d_{s}\left(\bigvee_{q=0}^{s}\left[g\left(t_{2}, q\right)-g\left(t_{1}, q\right)\right]\right) \\
& =\omega(a, \varepsilon)+\frac{\alpha}{\Gamma(\alpha)} \omega_{\|x\|}^{1}(f, \varepsilon) \bigvee_{s=0}^{t_{2}} g\left(t_{2}, s\right) \\
& +\frac{\alpha}{\Gamma(\alpha)} \phi(\|x\|) \bigvee_{s=t_{1}}^{t_{2}} g\left(t_{2}, s\right) \\
& +\frac{\alpha}{\Gamma(\alpha)} \phi(\|x\|) \bigvee_{s=0}^{t_{1}}\left[g\left(t_{2}, s\right)-g\left(t_{1}, s\right)\right] \\
& \leq \omega(a, \varepsilon)+\frac{\alpha}{\Gamma(\alpha)}\left[K \omega_{\|x\|}^{1}(f, \varepsilon)+\phi(\|x\|) N(\varepsilon)\right. \\
& +\phi(\|x\|) M(\varepsilon)],
\end{aligned}
$$

where we denoted

$$
\begin{gathered}
w_{r}^{1}(f, \varepsilon)=\sup \left\{\left|f\left(t_{2}, s, x\right)-f\left(t_{1}, s, x\right)\right|: t_{1}, t_{2} \in I\right. \\
\left.\left|t_{2}-t_{1}\right| \leq \varepsilon, x \in[-r, r]\right\}
\end{gathered}
$$

Further, let us observe that in view of the uniform continuity of the function $f$ on the set $\Delta \times[-\|x\|,\|x\|]$ we infer that $\omega_{\|x\|}^{1}(f, \varepsilon) \rightarrow 0$ as $\varepsilon \rightarrow 0$. Moreover, invoking assumption (i) and the properties of the functions $M(\varepsilon)$ and $N(\varepsilon)$ defined by (22), we conclude that the function $F x$ is continuous on the interval $I$. This means that the operator $F$ transforms $C(I)$ into itself.

In what follows we show that the operator $F$ is continuous on the space $C(I)$. To this end fix arbitrarily a number $\varepsilon>0$ 
and take $x, y \in C(I)$ such that $\|x-y\| \leq \varepsilon$. Then, in view of Lemmas 1 and 2, for an arbitrarily fixed $t \in I$ we obtain

$$
\begin{aligned}
& |(F x)(t)-(F y)(t)| \\
& \leq \frac{\alpha}{\Gamma(\alpha)} \mid \int_{0}^{t} s^{p} f(t, s, x(s)) d_{s} g(t, s) \\
& \quad-\int_{0}^{t} s^{p} f(t, s, y(s)) d_{s} g(t, s) \mid \\
& \leq \frac{\alpha}{\Gamma(\alpha)} \int_{0}^{t} s^{p}|f(t, s, x(s))-f(t, s, y(s))| d_{s} \\
& \quad \times\left(\bigvee_{q=0}^{s} g(t, q)\right) .
\end{aligned}
$$

Hence, denoting by $P$ the number

$$
P=\|x\|+\varepsilon
$$

and introducing the notation

$$
\begin{gathered}
\omega_{P}(f, \varepsilon)=\sup \{|f(t, s, x)-f(t, s, y)|:(t, s) \in \Delta, \\
x, y \in[-P, P],|x-y| \leq \varepsilon\},
\end{gathered}
$$

we obtain the following estimate:

$$
\begin{aligned}
|(F x)(t)-(F x)(t)| & \leq \frac{\alpha}{\Gamma(\alpha)} \omega_{P}(f, \varepsilon) \int_{0}^{t} s^{p} d_{s}\left(\bigvee_{q=0}^{s} g(t, q)\right) \\
& \leq \frac{\alpha}{\Gamma(\alpha)} \omega_{P}(f, \varepsilon) \int_{0}^{t} d_{s}\left(\bigvee_{q=0}^{s} g(t, q)\right) \\
& \leq \frac{\alpha}{\Gamma(\alpha)} \omega_{P}(f, \varepsilon) \bigvee_{s=0}^{t} g(t, s) \\
& \leq \frac{\alpha}{\Gamma(\alpha)} K \omega_{P}(f, \varepsilon) .
\end{aligned}
$$

Now, applying the fact that the function $f$ is uniformly continuous on the set $\Delta \times[-P, P]$, we conclude that $\omega_{P}(t, \varepsilon) \rightarrow$ 0 as $\varepsilon \rightarrow 0$. Combining this fact with estimate (27) we deduce that the operator $F$ is continuous on the space $C(I)$.

Further on, let us fix arbitrarily $x \in C(I)$. Then, applying Lemmas 1 and 2 and arguing as above, for $t \in I$, we obtain

$$
\begin{aligned}
|(F x)(t)| \leq & |a(t)|+\frac{\alpha}{\Gamma(\alpha)}\left|\int_{0}^{t} s^{p} f(t, s, x(s)) d_{s} g(t, s)\right| \\
\leq & \|a\| \\
& +\frac{\alpha}{\Gamma(\alpha)} \int_{0}^{t} s^{p}|f(t, s, x(s))| d_{s}\left(\bigvee_{q=0}^{s} g(t, q)\right) \\
< & \|a\|+\frac{\alpha}{\Gamma(\alpha)} \phi(\|x\|) \int_{0}^{t} d_{s}\left(\bigvee_{q=0}^{s} g(t, q)\right)
\end{aligned}
$$

$$
\begin{aligned}
& \leq\|a\|+\frac{\alpha}{\Gamma(\alpha)} \phi(\|x\|) \bigvee_{s=0}^{t} g(t, s) \\
& \leq\|a\|+\frac{\alpha}{\Gamma(\alpha)} K \phi(\|x\|) .
\end{aligned}
$$

Now, invoking assumption (vii) we deduce that there exists a number $r_{0}>0$ such that the operator $F$ transforms the ball $B_{r_{0}}$ into itself.

In what follows let us choose a number $\varepsilon>0$. Next, take arbitrary numbers $t_{1}, t_{2} \in I$ such that $t_{1}<t_{2}$ and $t_{2}-t_{1} \leq \varepsilon$. Then, for arbitrarily fixed element $x \in B_{r_{0}}$, in view of estimate (23), we obtain

$$
\begin{aligned}
& \left|(F x)\left(t_{2}\right)-(F x)\left(t_{1}\right)\right| \\
& \leq \omega(a, \varepsilon) \\
& \quad+\frac{\alpha}{\Gamma(\alpha)}\left[K \omega_{r_{0}}^{1}+\phi\left(r_{0}\right) N(\varepsilon)+\phi\left(r_{0}\right) M(\varepsilon)\right] .
\end{aligned}
$$

Then, based on the properties of the functions $\varepsilon \rightarrow \omega(a, \varepsilon)$, $\varepsilon \rightarrow \omega_{r_{0}}^{1}(f, \varepsilon), \varepsilon \rightarrow M(\varepsilon)$, and $\varepsilon \rightarrow N(\varepsilon)$, from (29) and the Arzéla-Ascoli criterion for relative compactness in the space $C(I)$, we conclude that the subset $F\left(B_{r_{0}}\right)$ of the ball $B_{r_{0}}$ is relatively compact in the space $C(I)$. Thus, applying the Schauder fixed point principle, we conclude that the operator $F$ has at least one fixed point $x$ belonging to the ball $B_{r_{0}}$. Obviously, the function $x=x(t)$ is a solution of (12) and the proof is complete.

\section{Further Discussions and an Example}

In this section we discuss, first of all, the applicability of assumption (iv). Observe that assumption (iv) seems to be not convenient to be used in a concrete situation. Indeed, we show that the mentioned assumption can be replaced by an assumption which is more convenient and is suitable for the use if we consider the Erdélyi-Kober integral equation (11).

Namely, we will utilize the following assumption.

(iv $^{\prime}$ ) For arbitrary $t_{1}, t_{2} \in I$ such that $t_{1}<t_{2}$ the function $s \rightarrow g\left(t_{2}, s\right)-g\left(t_{1}, s\right)$ is nonincreasing on the interval $\left[0, t_{1}\right]$. It can be shown [19] the following assertion.

Theorem 7. Let $g(t, s)=g: \Delta \rightarrow \mathbb{R}$. If the function $g$ satisfies assumptions (ii), (iv'), and (v), then $g$ satisfies assumption (iv).

Now, we focus on the study of the solvability of the Erdélyi-Kober integral equation (11). As we pointed out in Section 3, (11) is a special case of (12) if we assume that the function $g=g(t, s)$ has the form (13).

In what follows we show that the function $g(t, s)$ defined by (13) satisfies assumptions (ii) $-(v)$ formulated before Theorem 6. 
In order to prove this assertion, observe that it is rather obvious that the function $g$ defined by (13) satisfies assumptions (ii) and (v). To prove that $g$ satisfies (iii), let us notice that

$$
\frac{\partial g}{\partial s}=\alpha m s^{m-1}\left(t^{m}-s^{m}\right)^{\alpha-1}=\frac{\alpha m s^{m-1}}{\left(t^{m}-s^{m}\right)^{1-\alpha}}>0,
$$

for all $s \in[0, t)$. This means that the function $s \longrightarrow g(t, s)$ is nondecreasing on the interval $[0, t]$ and allows us to deduce that the function $g$ defined by (13) satisfies assumption (iii).

To prove that there is satisfied assumption (iv), fix arbitrary $t_{1}, t_{2} \in I$ such that $t_{1}<t_{2}$. Consider the function $G=G(s)$ defined by the formula

$$
\begin{aligned}
G(s) & =g\left(t_{2}, s\right)-g\left(t_{1}, s\right) \\
& =t_{2}^{\alpha m}-\left(t_{2}^{m}-s^{m}\right)^{\alpha}-t_{1}^{\alpha m}+\left(t_{1}^{m}-s^{m}\right)^{\alpha},
\end{aligned}
$$

for $s \in\left[0, t_{1}\right]$. Then we get

$$
\begin{aligned}
G^{\prime}(s) & =\alpha m s^{m-1}\left[\left(t_{2}^{m}-s^{m}\right)^{\alpha-1}-\left(t_{1}^{m}-s^{m}\right)^{\alpha-1}\right] \\
& =\alpha^{n} s^{m-1}\left[\frac{1}{\left(t_{2}^{m}-s^{m}\right)^{1-\alpha}}-\frac{1}{\left(t_{1}^{m}-s^{m}\right)^{1-\alpha}}\right] .
\end{aligned}
$$

It is easily seen that $G^{\prime}(s)<0$ for $s \in\left[0, t_{1}\right)$ which permits us to infer that the function $g=g(t, s)$ defined by (13) satisfies assumption (iv').

Summing up, we conclude that the function $g$ defined in (13) satisfies assumptions (ii)-(v).

On the basis of the above established facts we can formulate the following existence result concerning integral equation (11).

Theorem 8. Assume that there are satisfied assumptions (i) and (vi), and the following one.

(vii') There exists a positive solution $r_{0}$ of the inequality

$$
\|a\|+\frac{\alpha}{\Gamma(\alpha)} \phi(r) \leq r .
$$

Then (11) has at least one solution in the space $C(I)$ belonging to the ball $B_{r_{0}}$.

Indeed, the above theorem is an easy consequence of the above established facts concerning the function $g(t, s)$ defined by (13) and Theorem 6 as well as the equality $K=1$, where $K$ is the constant defined by (19).

Now, we illustrate our result contained in Theorem 8 by an example.

Example 9. Let us consider the following nonlinear integral equation of Erdélyi-Kober type:

$$
\begin{aligned}
x(t)= & t \exp t \\
& +\frac{1}{\Gamma(1 / 2)} \int_{0}^{t} \frac{(4 / 3) s^{7 / 3}\left(t+\sin s^{2}+\sqrt[3]{x^{2}(s)}\right)}{\left(t^{4 / 3}-s^{4 / 3}\right)^{1 / 2}} d s,
\end{aligned}
$$

for $t \in I=[0,1]$. Observe that this equation can be rewritten in the form of (11); that is,

$$
\begin{aligned}
x(t)= & t \exp t \\
& +\frac{1}{\Gamma(1 / 2)} \int_{0}^{t} \frac{(4 / 3) s^{1 / 3} s^{2}\left(t+\sin s^{2}+\sqrt[3]{x^{2}(s)}\right)}{\left(t^{4 / 3}-s^{4 / 3}\right)^{1 / 2}} d s .
\end{aligned}
$$

Notice that (35) is a particular case of (11) if we put $a(t)=$ $t \exp t, \alpha=1 / 2, m=4 / 3, p=2$, and

$$
f(t, s, x)=t+\sin s^{2}+x^{2 / 3} .
$$

Now we verify that the above indicated components of (35) satisfy the assumptions of Theorem 8 . Indeed, the function $a=a(t)$ is a member of the space $C(I)$ and $\|a\|=e$. Further, we have that $f$ is continuous on the set $\Delta \times \mathbb{R}$ and the following estimate holds for arbitrary $t, s \in \mathbb{R}_{+}$and $x \in \mathbb{R}$ :

$$
|f(t, s, x)| \leq t+\left|\sin s^{2}\right|+x^{2 / 3} \leq 2+x^{2 / 3} .
$$

This implies that the function $f$ satisfies assumption (vi) with the function $\phi(r)=2+r^{2 / 3}$ being nondecreasing on $\mathbb{R}_{+}$. Further, let us take into account inequality (33) which in our case has the form

$$
e+\frac{1}{2 \Gamma(1 / 2)}\left(2+r^{2 / 3}\right) \leq r
$$

or equivalently

$$
e+\frac{1}{2 \sqrt{\pi}}\left(2+r^{2 / 3}\right) \leq r .
$$

Let us write the above inequality in a more transparent form:

$$
e+\frac{1}{\sqrt{\pi}}+\frac{1}{2 \sqrt{\pi}} e^{2 / 3} \leq r .
$$

Then, it is easily seen that the number $r_{0}=4$ seems to be a "sufficiently" optimal solution of inequality (40).

On the basis of Theorem 8 we conclude that (35) has at least one solution in the space $C(I)$ belonging to the ball $B_{4}$.

Remark 10. It is worthwhile mentioning that the term $s^{p}$ appearing both in (11) and in (12) has no significance in our considerations and can be included in the function $f=$ $f(t, s, x)$. Nevertheless, we have separated that term since it will play certain role in our further study of nonlinear ErdélyiKober integral equations considered in spaces of functions defined on the half-axis $\mathbb{R}_{+}$.

Details will appear elsewhere.

\section{Conflict of Interests}

The authors declare that there is no conflict of interests regarding the publication of this paper. 


\section{References}

[1] K. Diethelm, The Analysis of Fractional Differential Equations, vol. 2004 of Lecture Notes in Mathematics, Springer, Berlin, Germany, 2010.

[2] A. A. Kilbas, H. M. Srivastava, and J. J. Trujillo, Theory and Applications of Fractional Differential Equations, vol. 204, Elsevier Science B.V., Amsterdam, The Netherlands, 2006.

[3] K. S. Miller and B. Ross, An Introduction to the Fractional Calculus and Fractional Differential Equations, John Wiley \& Sons, New York, NY, USA, 1993.

[4] I. Podlubny, Fractional Differential Equations, vol. 198, Academic Press, San Diego, Calif, USA, 1999.

[5] R. Hilfer, Applications of Fractional Calculus in Physics, World Scientific, River Edge, NJ, USA, 2000.

[6] V. Lakshmikantham, S. Leela, and J. Vasundara Devi, Theory of Fractional Dynamic Systems, Cambridge Scientific Publishers, Cambridge, UK, 2009.

[7] S. G. Samko, A. A. Kilbas, and O. I. Marichev, Fractional Integrals and Derivatives, Gordon and Breach Science, Amsterdam, The Netherlands, 1993.

[8] H. M. Srivastava and R. K. Saxena, "Operators of fractional integration and their applications," Applied Mathematics and Computation, vol. 118, no. 1, pp. 1-52, 2001.

[9] J. A. Alamo and J. Rodríguez, "Operational calculus for modified Erdélyi-Kober operators," Serdica Bulgaricae Mathematicae Publicationes, vol. 20, no. 3-4, pp. 351-363, 1994.

[10] H. H. Hashem and M. S. Zaki, "Carathéodory theorem for quadratic integral equations of Erdélyi-Kober type," Journal of Fractional Calculus and Applications, vol. 4, no. 1, pp. 56-72, 2013.

[11] M. A. Darwish and K. Sadarangani, "On Erdélyi-Kober type quadratic integral equation with linear modification of the argument," Applied Mathematisc and Computation, vol. 238, pp. 30-42, 2014.

[12] J. R. Wang, C. Zhu, and M. Feckan, "Solvability of fully nonlinear functional equations involving Erdélyi-Kober fractional integrals on the unbounded interval," Optimization, 2014.

[13] J. Appell, J. Banaś, and N. Merentes, Bounded Variation and Around, vol. 17 of De Gruyter Series in Nonlinear Analysis and Applications, Walter de Gruyter, Berlin, Germany, 2014.

[14] I. P. Natanson, Theory of Functions of a Real Variable, Ungar, New York, NY, USA, 1960.

[15] N. Danford and J. T. Schwartz, Linear Operators, International Publishing, Leyden, The Netherlands, 1963.

[16] A. Erdélyi, "On fractional integration and its application to the theory of Hankel transforms," The Quarterly Journal of Mathematics, vol. 11, p. 293-303, 1940.

[17] A. Erdélyi and H. Kober, "Some remarks on Hankel transforms," The Quarterly Journal of Mathematics, vol. 11, pp. 212-221, 1940.

[18] H. Kober, "On fractional integrals and derivatives," The Quarterly Journal of Mathematics, vol. 11, pp. 193-211, 1940.

[19] J. Banaś and T. Zając, "A new approach to the theory of functional integral equations of fractional order," Journal of Mathematical Analysis and Applications, vol. 375, no. 2, pp. 375387, 2011.

[20] T. Zając, "Solvability of fractional integral equations on an unbounded interval through the theory of Volterra-Stieltjes integral equations," Zeitschrift für Analysis und ihre Anwendungen, vol. 33, no. 1, pp. 65-85, 2014. 


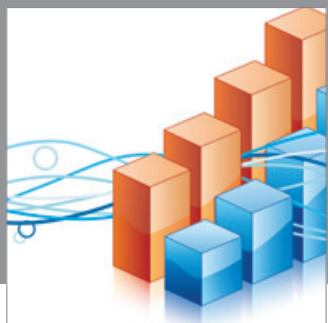

Advances in

Operations Research

mansans

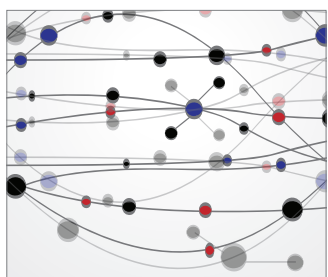

The Scientific World Journal
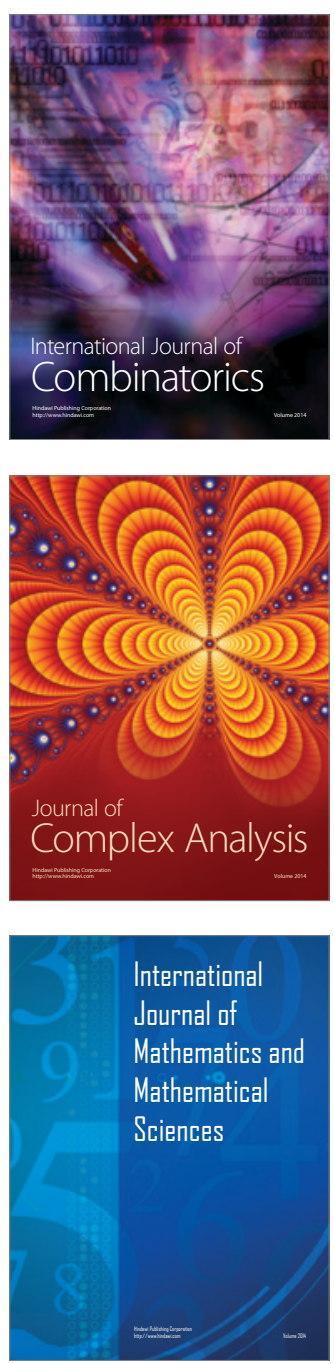
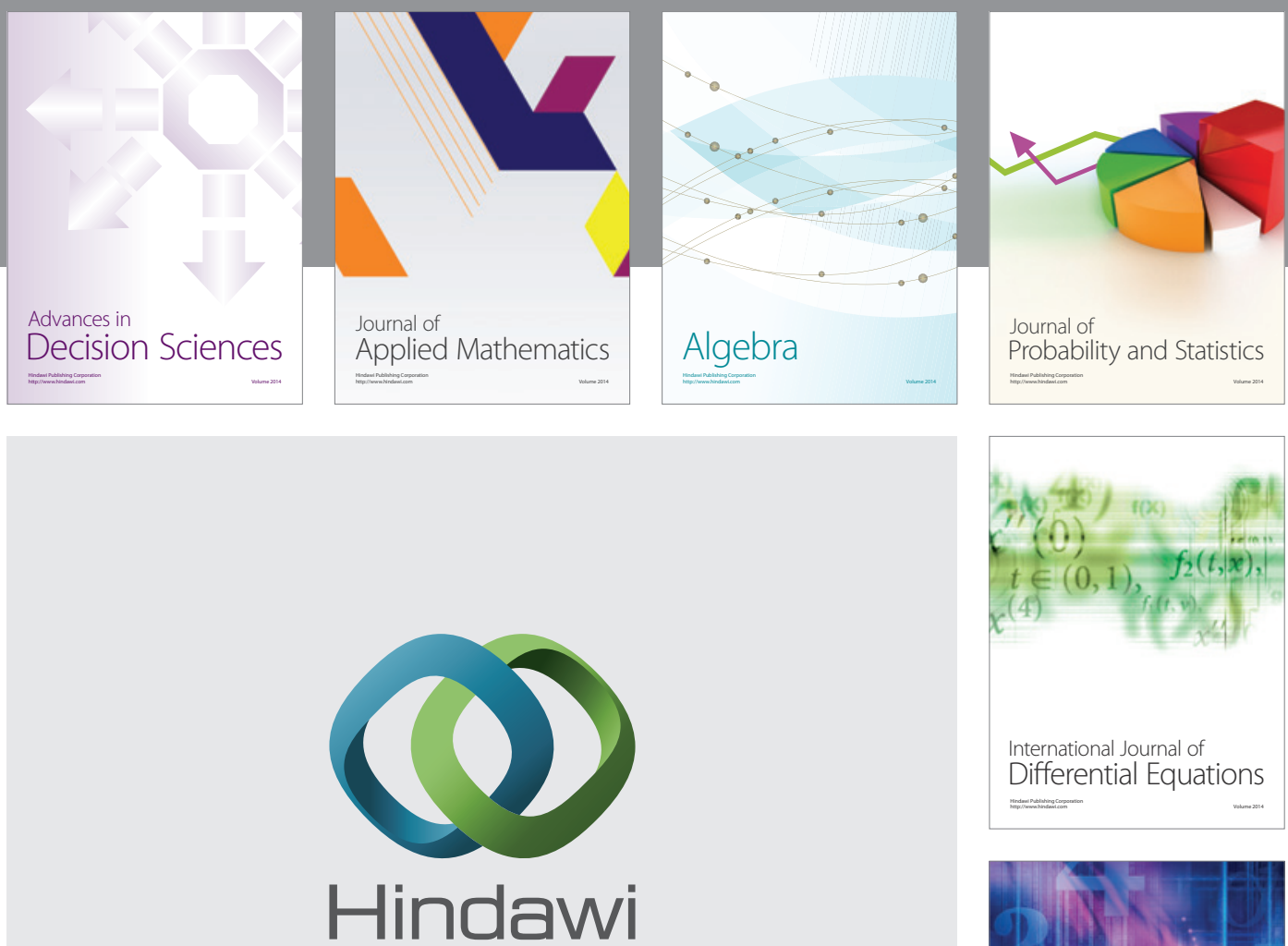

Submit your manuscripts at http://www.hindawi.com
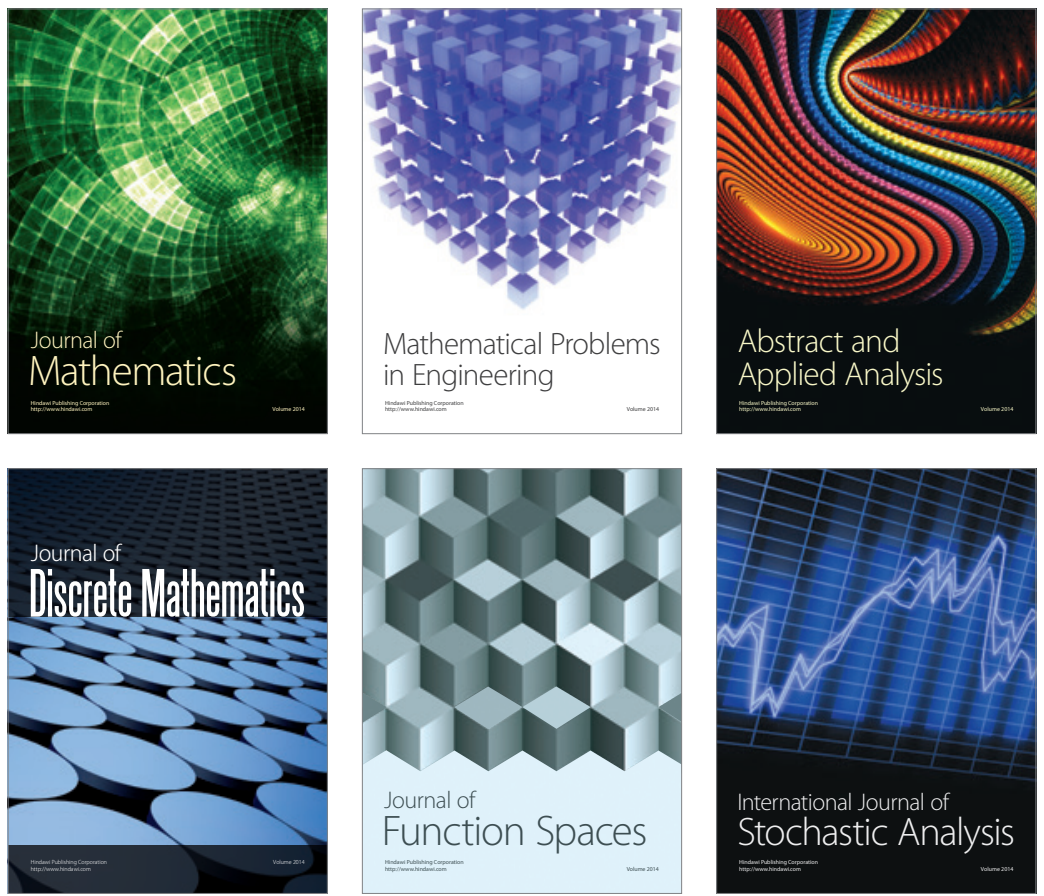

Journal of

Function Spaces

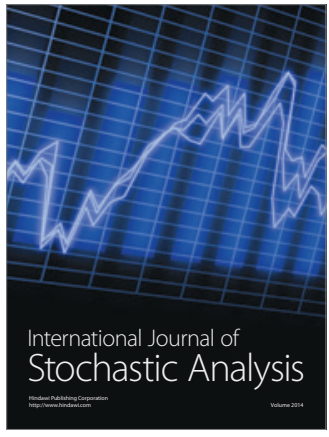

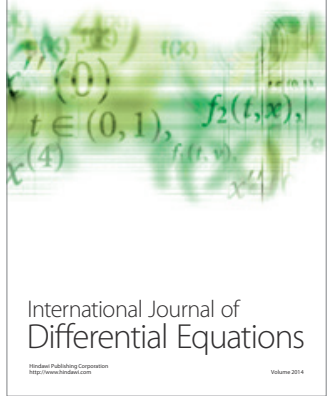
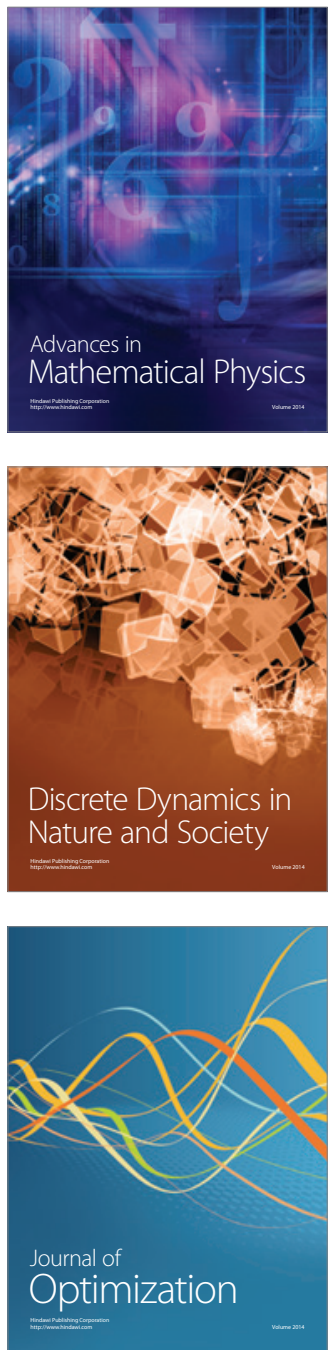